\section{Willingness to pay for dentin regeneration in a sample of dentate adults}

Birch S, Sohn W, Ismail AI, Lepkowski JM, Belli RF. Willingness to pay for dentin regeneration in a sample of dentate adults. Community Dent Oral Epidemiol 2004; 32: 210-6. (C) Blackwell Munksgaard, 2004

Abstract - Objectives: Measurement of individuals' valuation of dental treatments is important in the evaluation of new technologies. In this paper the value of dentin regeneration, a new treatment for teeth with reversible pulpitis, is measured based on what individuals say they would be willing to pay to receive the treatment. Methods: A total of 611 randomly selected dentate adults answered willingness to pay (WTP) and dental insurance questions. Detailed descriptions of the process and expected outcomes for dentin regeneration were presented to subjects as part of a larger study measuring preferences for different treatments. WTP was determined for two different levels of success for dentin regeneration. Results: At a success rate of 95\%, the mean WTP for dentin regeneration was $\$ 262.70$ (noninsured) and $\$ 11.00$ per month (insured subjects). For success rate of $75 \%$, the corresponding values were $\$ 210.90$ and $\$ 9.20$ per month. Multivariate analyses were used to identify any significant relationships between WTP and a range of variables covering socio-demographic, socioeconomic, dental experience and oral health status variables. The findings indicate that individuals' valuations of treatments involve substantial unexplained variation. About half of the noninsured subjects would pay for dentin regeneration if it cost $\$ 200$ per tooth. Conclusions: The data on the WTP for dentin regeneration indicate that a substantial percentage of adults will pay for this new technology. This study provides for the first time an estimate of WTP for dentin regeneration among the population.
Stephen Birch ${ }^{1}$, Woosung Sohn ${ }^{2}$, Amid I. Ismail ${ }^{2}$, James M. Lepkowski ${ }^{3}$ and Robert F. Belli ${ }^{4}$

${ }^{1}$ Department of Clinical Epidemiology and Biostatistics, Centre for Health Economics and Policy Analysis, McMaster University, Hamilton, Ontario, Canada, ${ }^{2}$ School of Dentistry, University of Michigan, Ann Arbor, MI, USA, ${ }^{3}$ Institute for Social Research, Ann Arbor, MI, USA, ${ }^{4}$ Department of Psychology, University of Nebraska, Lincoln, NE, USA
Key words: decision making; dental enamel proteins; endodontics; quality of life; regeneration; tooth extraction

Stephen Birch, Department of Clinical Epidemiology and Biostatistics, Centre for Health Economics and Policy Analysis, McMaster University, 1200, Main Street West, Hamilton, Ontario L8N 3Z5, Canada Tel: 905-525-9140 x23244

Fax: 905-546-5211

e-mail: birch@mcmaster.ca

Submitted 14 April 2003; accepted 18 December 2003
The development of new technologies provides an increasing range of possibilities for the treatment of dental problems. Clinical evaluations determine whether the new technology can work (efficacy) and does work (effectiveness) (1). However, acceptance and adoption by patients and the general public will depend on their valuation of the procedure. For example, a new treatment might be found to be more effective than the existing treatment for the same condition. But if the new treatment involves more time, discomfort or inconvenience for the patient, its use in place of the existing treatment might lead to a reduction in the patient's well-being. Patient uptake and compliance may be problematic where new treatments are introduced based on the evaluation of clinical outcomes alone.
The measurement of the value of new technologies to individuals is an important part of evaluation (2).

This paper presents data on willingness to pay (WTP) for dentin regeneration by adults residing in the Detroit Metropolitan area (Wayne, Oakland and Macomb counties), Michigan. Dentin regeneration is a new procedure used to save teeth with reversible pulpitis. The treatment involves removal of the damaged part of the dental pulp. A dentin regeneration protein (e.g. TGF- $\beta$, BMP-2,-4 and -7) is placed on the live pulp to stimulate the growth of new dentin and the retention of a 'live' tooth.

Several methods have been used for measuring individuals' valuations of health care interventions including the time trade-off (TTO) (3-5), and the standard gamble (SG) (6-8). These measures are 
used to express the individuals' valuations in a way that is meaningful to a health care decision maker [e.g. quality adjusted life years (QALYs), quality adjusted tooth years (QATYs) or healthy years equivalent (HYEs)] $(9,10)$. These approaches require individuals to express their valuations in terms of changes in their expected length of (tooth) life or risk of (tooth) survival and can be helpful in determining relative preferences among different interventions. However, individual values measured using these nonmonetary approaches are not directly comparable with the costs of the treatment (11). In order to assess whether a particular intervention is worthwhile per se, from the perspective of the individual, individual valuations are measured by what individuals say they would be WTP, either in terms of the out of pocket payments to receive the intervention or the additional insurance payments to have the treatment covered under insurance (12-17). The conceptual basis of the WTP approach together with a summary of its application in health services research has been provided elsewhere (18).

\section{Methods}

The data were collected as a part of the Dentin Regeneration Preference Project (DRPP), which aimed to measure the preferences of adults for various dental treatments including a new technology - dentin regeneration. Data collection was based on a cross-sectional survey that incorporated a sample representing a population of 3 million adults residing in the Detroit tri-county area and carried out between August 2000 and August 2001. Data collection methods comprised of a computeraided home interview and a self-administered questionnaire. The conduct of this research was approved by the University of Michigan IRB for Health Sciences. Details on the sampling and measurement methods are described in another paper (19). A summary is included here.

\section{Sampling and recruitment process}

The sampling was based on a list-assisted random digit dialling protocol. Random digit dialling has been demonstrated to result in representative samples (20). In Michigan, the Federal Communication Commission reports that less than $5 \%$ of households do not have a telephone. In 1996, 85\% of residents in the USA with an income of less than $\$ 10000$ per year have a telephone at home.
A list of randomly generated telephone numbers was obtained from Genesys Inc. (Philadelphia, PA, USA). Numbers were selected from banks of 100 consecutive telephone numbers defined by area code and prefix combinations for the tri-county area with one or more telephones. A sample of 2372 numbers was randomly selected and screened to eliminate nonresidential telephones.

Telephone screening was carried out by trained interviewers of the Institute for Social Research, University of Michigan, using the Computer-Assisted Telephone Interviewing (CATI) system. After exclusion of nonresidential numbers a short questionnaire was administered to determine eligibility and dental insurance coverage (not including Medicaid) of each individual in the household. Eligibility criteria for participants were: (i) adults aged 18-69 years, (ii) currently live in the household, and (iii) with at least one natural tooth in the mouth. Households were categorized as 'insured' if the respondent reported that all adults in the household were covered by a dental insurance plan not including Medicaid. This enabled oversampling of noninsured households (i.e. households where at least one adult was not covered by a dental insurance plan) based on oral health survey data for the tri-county area that found $70 \%$ of subjects had dental insurance (21). All noninsured households were invited to participate in the study. In households where all adults have dental insurance, the CATI system randomly selected one-half of such households to participate. Once a household was selected for study, all eligible adults in the household were invited to participate in the study.

Individuals were asked to suppose that they had a decayed tooth with a deep cavity. Descriptions were provided in words and pictures of the procedures and expected outcomes of four different treatments - filling the tooth, root canal therapy, extraction and dentin regeneration. Each subject was asked if he/she would consider the dentin regeneration treatment. Subjects who reported that they would consider the treatment were asked how much they would be willing to pay for dentin regeneration. The precise question differed according to whether subjects reported having dental insurance or not. Those without insurance were asked the dollar amount they would be willing to pay out of pocket for the treatment. Subjects with insurance were asked the amount of additional premium they would be willing to pay per month to have their insurance cover extended to include dentin regeneration. 
Because the success rate of dentin regeneration is unknown at this stage the questions were asked for both $75 \%$ and $95 \%$ success rates. These rates were selected to be less than and equal to the expected success rate of root canal therapy (22), respectively. Mean WTP (noninsured subjects only) and mean WTP increased monthly premiums (insured subjects only) were calculated for each success rate. The validity of the WTP question was considered by comparing responses for the different success rates. A higher success rate was expected to be associated with higher levels of WTP.

The test-retest reliability of the WTP scores was evaluated using a sample of 40 adults. The Pearson's correlation coefficient in the test-retest pilot study was 0.96 and there were no statistically or clinically significant differences in the mean WTP values.

Bivariate analyses were performed on a series of sociodemographic and socioeconomic characteristics, oral health status measures and dental care experience. It may be that there is a high level of correlation between some of the background characteristics (e.g. education and income). Multiple regression analysis was used to estimate the independent effect of individual variables after controlling for the influence of other variables. All background characteristics were entered into the multiple regression equation together with those oral health and dental care experience variables found to be significant in the bivariate analysis of either the insured or noninsured. As described elsewhere (19), the sampling method resulted in a disproportionate and clustered sample; hence, we used SUDAAN to analyse the data to account for clustering effects of individuals within households. All percentages and means presented in this paper are weighted.

\section{Results}

In total 611 subjects completed the WTP questions, 380 (72.6\%: a weighted percentage reflecting the population estimate of insured adults in the area) of these had some form of dental insurance. Table 1 reports on the percentage considering having the procedure and the mean WTP for the procedure (or for additional premiums) of those subjects. Separate results are reported for different success rates. The percentage considering having the procedure and the mean WTP increase with the success rate in both insured and noninsured groups. Mean differences in WTP between the higher and lower levels of success are positive and significantly different to zero for both groups. At the individual level only $1.7 \%$ of insured subjects and $3.2 \%$ of noninsured subjects expressed WTP amounts that decreased as the success rate increased suggesting that only a very small proportion of subjects misunderstood the WTP exercise. In the rest of this section only results for the noninsured population are presented. The results for the insured population are available from the authors and were generally the same unless otherwise indicated (see Discussion).

Figure 1 presents the demand curve for dentin regeneration for both levels of success. This shows the percentage of subjects that would pay for dentin regeneration at different prices for the procedure. At prices above $\$ 400$, around $25 \%$ of subjects are willing to pay for the procedure (95\% success rate). Changes in the price above $\$ 400$ have only small effects on the percentage of subjects willing to pay the price. However, reductions in price below $\$ 400$ lead to greater responsiveness in demand with approximately $50 \%$ of subjects willing to pay $\$ 200$ for the procedure. The demand curve at the lower level of success rate $(75 \%)$ lies below the $95 \%$ success rate curve indicating that at any price level, higher success rates are associated with greater demand for the procedure.

Table 2 reports the mean WTP for the intervention for the $95 \%$ success level by background characteristics, oral health status and dental care experience among noninsured subjects who would consider having dentin regeneration. The observed

Table 1. Percentage of subjects that would consider dentin regeneration (DR) and mean willingness to pay (WTP) for DR by insurance status and level of success

\begin{tabular}{|c|c|c|c|c|c|c|c|}
\hline \multicolumn{2}{|c|}{ Success rate of DR (\%) } & \multicolumn{2}{|c|}{$\begin{array}{l}\text { Percentage considering } \\
\text { DR } \\
\end{array}$} & \multicolumn{2}{|l|}{ Mean WTP (\$) } & \multicolumn{2}{|c|}{$\begin{array}{l}\text { Difference in WTP } \\
(95 \%-75 \%)\end{array}$} \\
\hline Insurance status & $n^{\mathrm{a}}$ & $95 \%(\mathrm{SE})$ & $75 \%(\mathrm{SE})$ & $95 \%$ mean $(\mathrm{SE})$ & $75 \%$ mean $(\mathrm{SE})$ & Mean (SE) & $P$-value \\
\hline Noninsured & 231 & $96.8(1.1)$ & $88.0(2.5)$ & $262.7(16.4)$ & $210.9(14.9)$ & $53.9(6.1)$ & $<0.0001$ \\
\hline Insured & 380 & $99.1(0.5)$ & $96.8(1.1)$ & $11.0(0.8)$ & $9.2(0.9)$ & $2.3(0.3)$ & $<0.0001$ \\
\hline
\end{tabular}

${ }^{\mathrm{a}}$ Total number of subjects in the sample $=611$. 


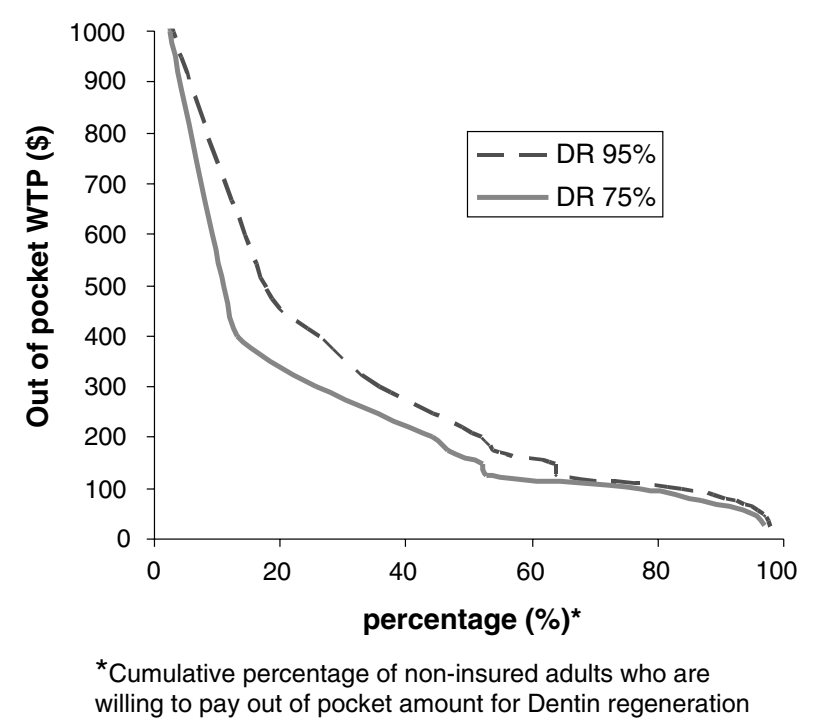

Fig. 1. Demand curve for detain regeneration among noninsured adults.

patterns are as expected with higher WTP being associated with younger age groups, higher education higher incomes, whites, better oral health and lower perceptions of need for treatment. The differences in WTP were statistically significant $(P<0.05)$ only for perceived need of extraction and regular dental visits.

Table 3 records the results of the multiple regression equation for WTP with a 95\% success rate. The patterns observed in the bivariate analyses remain with the exception of race with the mean WTP of African-Americans and other nonwhites being higher than whites. However, only the regular dentist visit is statistically significant with those having regular visits also having a higher mean WTP.

\section{Discussion}

Improvements in technology provide new ways of dealing with illness and diseases. However, the costs of new technologies mean that other things have to be given up in order to support them. The literature on the valuation of health outcomes has been dominated by methods of valuation that involve trade-offs between the quantity and quality of life years or tooth years. Although these methods can provide helpful ways of measuring relative preferences among different interventions, in the real world access to health services is not determined by individuals' willingness to forgo the risk to or quantity of future life years. Instead, access to
Table 2. Willingness to pay for dentin regeneration by background characteristics (US\$)

\begin{tabular}{|c|c|c|c|}
\hline Characteristics & $n$ & $\operatorname{Mean}^{\mathrm{a}}$ (SE) & $P$-value ${ }^{\mathrm{b}}$ \\
\hline \multicolumn{4}{|l|}{ Sex } \\
\hline Male & 97 & $265.8(23.5)$ & \multirow[t]{2}{*}{0.8479} \\
\hline Female & 124 & $259.4(23.5)$ & \\
\hline \multicolumn{4}{|l|}{ Age (years) } \\
\hline$<30$ & 69 & $261.0(29.3)$ & \multirow[t]{3}{*}{0.6118} \\
\hline $30-49$ & 100 & $277.7(25.6)$ & \\
\hline$>50$ & 52 & $236.0(32.8)$ & \\
\hline \multicolumn{4}{|l|}{ Race/ethnicity } \\
\hline Whites & 140 & $278.8(20.9)$ & \multirow[t]{3}{*}{0.0841} \\
\hline African-Americans & 52 & $205.9(27.7)$ & \\
\hline Others & 29 & 289.5 (49.9) & \\
\hline \multicolumn{4}{|l|}{ Education } \\
\hline$>12$ & 112 & $301.8(23.4)$ & \multirow[t]{3}{*}{0.0719} \\
\hline 12 & 56 & $234.2(32.9)$ & \\
\hline$<12$ & 47 & $216.5(33.8)$ & \\
\hline \multicolumn{4}{|l|}{ Annual income ${ }^{c}$} \\
\hline$>60$ & 39 & $337.5(28.2)$ & \multirow[t]{4}{*}{0.0522} \\
\hline $40-60$ & 33 & $304.9(33.5)$ & \\
\hline $20-40$ & 51 & $207.6(42.5)$ & \\
\hline$\leq 20$ & 72 & $226.5(37.9)$ & \\
\hline \multicolumn{4}{|c|}{ Self-perceived oral health status } \\
\hline Excellent/very good & 57 & $255.7(24.4)$ & \multirow[t]{3}{*}{0.5011} \\
\hline Good & 73 & $292.1(29.1)$ & \\
\hline Fair to poor & 88 & $247.0(28.5)$ & \\
\hline \multicolumn{4}{|l|}{ Perceived need of fillings } \\
\hline Yes & 70 & $248.4(25.1)$ & \multirow[t]{2}{*}{0.4600} \\
\hline No & 148 & $272.8(21.3)$ & \\
\hline \multicolumn{4}{|c|}{ Perceived need of tooth extraction } \\
\hline Yes & 38 & $196.4(27.1)$ & \multirow[t]{2}{*}{0.0127} \\
\hline No & 180 & $279.7(19.1)$ & \\
\hline \multicolumn{4}{|c|}{ Had tooth pain in last 30 days } \\
\hline Yes & 62 & $222.7(32.3)$ & \multirow[t]{2}{*}{0.1259} \\
\hline No & 157 & $280.9(19.5)$ & \\
\hline \multicolumn{4}{|l|}{ Ever had extraction } \\
\hline Yes & 155 & $263.0(19.9)$ & \multirow[t]{2}{*}{0.8510} \\
\hline No & 62 & $270.1(31.6)$ & \\
\hline \multicolumn{4}{|c|}{ Ever had root canal therapy } \\
\hline Yes & 80 & $289.2(30.3)$ & \multirow[t]{2}{*}{0.2833} \\
\hline No & 138 & $251.2(18.9)$ & \\
\hline \multicolumn{4}{|l|}{ Ever had fillings } \\
\hline Yes & 199 & $275.3(17.6)$ & \multirow[t]{2}{*}{0.0647} \\
\hline No & 20 & $183.5(44.7)$ & \\
\hline Visit dentist regularly & & & \\
\hline Yes & 88 & $316.0(29.6)$ & 0.0150 \\
\hline No & 130 & $231.3(18.2)$ & \\
\hline
\end{tabular}

${ }^{\mathrm{a}}$ The percentages and mean values are weighted.

${ }^{\mathrm{b}} P$-value from $t$-test or ANOVA.

'Income expressed in 1000 dollars.

a particular health service will depend upon whether the individual has a means of paying for the service and, if so, how much he or she is willing to pay for that service.

In this paper, we used a WTP instrument to measure individuals' valuation of dentin regeneration. There appeared to be widespread understanding of the measurement procedure with only a small minority of subjects providing responses 
Table 3. Linear regression of willingness to pay for dentin regeneration among noninsured subjects

\begin{tabular}{|c|c|c|}
\hline & $\beta(\mathrm{SE})$ & $P$-value ${ }^{a}$ \\
\hline \multicolumn{3}{|l|}{ Sex (ref: female) } \\
\hline Male & $40.3(34.0)$ & 0.2373 \\
\hline \multicolumn{3}{|l|}{ Age (ref: <30) } \\
\hline $30-50$ & $2.1(40.7)$ & 0.4684 \\
\hline$\geq 50$ & $-46.6(41.2)$ & \\
\hline \multicolumn{3}{|c|}{ Race/ethnicity (ref: white) } \\
\hline African-Americans & $18.4(40.1)$ & 0.8047 \\
\hline Others & $38.8(60.6)$ & \\
\hline \multicolumn{3}{|c|}{ Education (ref: <12 years) } \\
\hline 12 years & $51.4(52.0)$ & 0.3073 \\
\hline$>12$ years & $79.1(51.4)$ & \\
\hline \multicolumn{3}{|c|}{ Annual income ${ }^{b}$ (ref: $\left.<20\right)$} \\
\hline $20-40$ & $-42.2(36.3)$ & 0.1490 \\
\hline $40-60$ & $65.8(67.7)$ & \\
\hline$>60$ & $69.2(65.5)$ & \\
\hline \multicolumn{3}{|c|}{ Regular dental visit (ref: no) } \\
\hline Yes & $95.2(44.7)$ & 0.0341 \\
\hline \multicolumn{3}{|l|}{ Need of filling (ref: no) } \\
\hline Yes & $4.1(30.6)$ & 0.8943 \\
\hline \multicolumn{3}{|c|}{ Need of extraction (ref: no) } \\
\hline Yes & $-48.1(39.2)$ & 0.2207 \\
\hline \multicolumn{3}{|c|}{ Ever had tooth filling (ref: no) } \\
\hline Yes & $55.8(52.9)$ & 0.2943 \\
\hline \multicolumn{3}{|c|}{ Oral health (ref: excellent/very good) } \\
\hline Good & $107.2(48.1)$ & 0.0822 \\
\hline Fair/poor & $111.1(60.1)$ & \\
\hline$R^{2}$ & 0.11 & \\
\hline
\end{tabular}

${ }^{\text {a }} P$-value for Wald test.

${ }^{\mathrm{b}}$ Income expressed in 1000 dollars.

that indicated inconsistency (i.e. WTP increased as success rate decreased). Subjects reported being willing to pay in order to have access to dentin regeneration. However, there was substantial variation among subjects in their reported WTP and our attempts to identify the determinants of WTP met with only modest success. How people value the potential benefits of the technology together with the purchasing power they are willing to forgo in return for these benefits involves substantial unexplained variation.

Some interesting patterns in WTP are observed in terms of the socioeconomic and sociodemographic characteristics. For example, females and older persons are willing to pay less on average than males and younger persons among the noninsured, although these relationships are statistically insignificant. It is worth noting that these patterns were reversed among noninsured subjects. This might reflect the selection of individuals into insurance, with women, older persons and those with higher education being more risk averse on average and hence having a greater demand for the risk-sharing features of insurance. In other words, the non-random nature of the distribution of insurance might be influencing the association between WTP and each of these variables.

The association with income is in accordance with expectations with those able to pay more (i.e. higher income groups) having higher WTP. In the case of race, African-Americans and other (nonwhites) adults have higher WTP than whites. The differences remain after controlling for variations in other explanatory variables (e.g. income, education, regular dental visits) but only in the case of insured individuals are statistically significant.

'Regular dental visits' was a behaviour that was positively correlated with WTP. Only the perceived need for, and experience of extractions was negatively correlated with WTP. In other words, individuals who appear to give a higher priority to oral health, as measured by their utilization of restorative services and levels of oral health, are willing to pay more for the new restorative procedure than individuals giving lower priority to oral health.

It is worth noting that among insured subjects, those perceiving the need for extractions and those not visiting the dentist regularly, both indicators of poor attitudes towards oral health, had higher WTP additional monthly premium than those without perceived need for extractions, and those visiting the dentist regularly. It may be that this reflects systematic variation in insurance provisions within the insured group. In this study, subjects were simply asked whether they were covered by a dental insurance plan (excluding Medicaid). No attempt was made to analyse differences in insurance arrangements, such as copayments, within the insured group. The WTP increased premiums might be associated with the level of current premiums, the levels of copayment or the provisions for coverage for alternative interventions for example. Variation in insurance arrangements might therefore be an important consideration in future research on WTP.

In terms of the level and distribution of WTP found in this study, the results are consistent with previous studies of WTP in dental care. For example, Matthews et al. (14) measured WTP increased premiums for alternative treatments for periodontal disease among a sample of patients with the condition and a sample of faculty and staff at a dental school. Among those subjects preferring deep cleaning to periodontal surgery WTP increased premiums ranged from $\$ 17.50$ to $\$ 22.50$ per month (Canadian dollars) for deep cleaning. In 
comparison among those subjects preferring surgery, WTP ranged from $\$ 26.66$ to $\$ 40.00$ per month. Sample size was insufficient to perform multivariate analysis to explain variations in WTP. However, there was a consistent pattern of increasing WTP with income among subjects preferring surgery.

As in the present study, attempts to explain variations in WTP have met with limited success. For example, in a study of WTP for a dental anaesthetic gel used in periodontal treatment, Matthews et al. (15) asked subjects in the general population as well as a patient sample both WTP out of pocket and WTP increased premiums. The mean WTP out of pocket was \$22.56 among patients and $\$ 16.67$ among the general population (Canadian dollars). The corresponding mean WTP increased premiums per month were $\$ 4.05$ (patients) and $\$ 3.28$ (general population). Only anxiety about needles and concern about dental pain were found to significantly explain variations in WTP in a multivariate analysis that included variables covering sociodemographic characteristics and dental care experience.

Shackley et al. (16) used WTP to measure the strength of preference for water fluoridation among the general population of Sheffield, UK, a city with relatively low naturally occurring fluoride in its water supply. Among those individuals' favouring water fluoridation the mean WTP additional taxes to support the programme was 12.63 UK pounds per year. In a multivariate analysis of variation in WTP only income was found to be significant with higher income groups having higher WTP on average.

Finally, Cunningham et al. (17) measured WTP for orthognathic treatment among patients with dentofacial deformity attending a hospital in London, UK. Unlike the other studies the WTP question was presented in terms of WTP to have the condition corrected as opposed to WTP for a treatment that has a probability of success. Among 40 adult patients with the condition, mean WTP was 6833 UK pounds. Although this figure is considerably higher than the WTP estimates of the current study as well as the studies by Matthews et al. (19-20), this may be because of the severity of the condition concerned and the framing of the WTP question around certain relief from the condition.

Comparison of the estimated value of the intervention with the opportunity cost of the resources used to provide the intervention determines whe- ther the intervention represents an efficient use of resources. It was not the intention of the current study to estimate the opportunity cost of dentin regeneration. Costs tend to change rapidly as new technologies are refined and diffused and differ according to the particular context in which the technology is introduced (23). Nevertheless, the estimated values provide important information to decision makers as they consider at what stage of development the technology becomes worthwhile from an economics perspective.

These findings have important implications for the provision of dental care. In particular, we should not expect that new technologies necessarily will be embraced by entire populations or all members of defined population groups, even where these technologies offer clear clinical advantages over existing treatments. Instead, an individual's overall best interests will depend on the balance between the individual's valuation of the benefits offered by the technology and the individuals' valuation of what they have to forgo to receive the treatment. WTP can be used to estimate these valuations and determine the expected demand for new technologies and for extended coverage for technologies under insurance plans and other prepayment arrangements for groups of subjects with different characteristics.

\section{Acknowledgements}

This study was funded by grant number DE13202-02, NIDCR/NIH/USPHS. We are grateful for the constructive comments and suggestions of two anonymous referees.

\section{References}

1. Bader J, Ismail A, Clarkson J. Evidence-based dentistry and the dental research community. J Dent Res 1999;78:1480-3.

2. Birch S, Ismail A. Patient preferences and the measurement of utilities in the evaluation of dental technologies. J Dent Res 2002;81:446-50.

3. Fyffe H, Deery C, Nugent Z, Nuttall N, Pitts N. The reliability of two methods of utility assessment in dentistry. Community Dent Health 1999;16:72-9.

4. Cohen M, Arthur J, Rodden J. Patients' retrospective preferences for extraction of asymptomatic third molars. Community Dent Health 1990;7:260-3.

5. Tulloch J, Antczak-Bouckoms A, Ung G. Evaluation of the costs and relative effectiveness of alternative strategies for the removal of mandibular third molars. Int J Technol Asses Health Care 1990;6:50515. 
6. Fyffe H, Kay E. Assessment of dental health state utilities. Community Dent Oral Epidemiol 1992;20:269-73.

7. Downer M, Julien J, Speight P. An interim determination of health gain form oral cancer and precancer screening: 1. Obtaining health state utilities. Community Dent Health 1997;14:139-42.

8. Birch S, Gafni A, Markham B, Marriott M, Lewis D, Main P. Health years equivalents as a measure of preferences for dental interventions. Community Dent Health 1998;15:233-42.

9. Gold M, Siegel J, Russell L, Weinstein M. Cost effectiveness in health and medicine. Oxford: Oxford University Press; 1996.

10. Birch S. Measuring dental health: an improvement on the DMF index. Community Dent Health 1986;3:303-11.

11. Drummond M, O'Brien B, Stoddart G, Torrance G. Methods for the economic evaluation of health care programs. Oxford: Oxford University Press; 1996.

12. Gafni A. Willingness to pay as a measure of benefits. Relevant questions in the context of public decision making about health care programs. Med Care 1991;29:1246-52.

13. Birch S, Gafni A, O'Brien B. Willingness to pay and the valuation of programs for the prevention and control of influenza. Pharmacoeconomics 1999;16: 55-61.

14. Matthews D, Gafni A, Birch S, Di Censo A. Willingness to pay for dental therapy: Development and testing of an instrument. J Public Health Dent 1999;59:44-51.
15. Matthews D, Rocchi A, Gafni A. Putting your money where your mouth is: willingness to pay for dental gel. Pharmacoeconomics 2002;20:245-55.

16. Shackley $\mathrm{P}$, Dixon S. Using contingent valuation to elicit preferences for water fluoridation. Applied Econ 2000;32:777-87.

17. Cunningham $S$, Hunt N. Relationship between utility values and willingness to pay in patients undergoing orthognathic treatment. Community Dent Health 2000;17:92-96.

18. Diener A, O'Brien B, Gafni A. Health care contingent valuation studies: a review and classification of the literature. Health Econ 1998;7:313-26.

19. Ismail AI, Sohn W, Birch S, Lepkowski JM, Belli R. Utilities of dentin regeneration among insured and uninsured adults. Community Dent Oral Epidemiol 2004;32:55-66.

20. Brick JM, Waksberg J, Kulp D, Starer A. Bias in listassisted telephone samples. Public Opinion Quarterly 1995;59:218-35.

21. Lang W, Borgnake W, Taylor G, Woolfolk M, Ronis D, Nyquist L. Evaluation and use of an index of oral health status. J Public Health Dent 1997;57:233-42.

22. Lazarski M, Walker W, Flores C, Schindler W, Hargreaves K. Epidemiological evaluation of the outcomes of non-surgical root canal treatment in a large cohort of insured dental patients. J Endo 2001;27:791-6.

23. Birch S, Gafni A. Economics and the evaluation of health care programmes: generalisability of methods and implications for generalisability of results. Health Policy 2003;64:207-19. 